\title{
Hypertension A Silent Epidemic in Nigeria: The Way Forward
}

\author{
Dalhatu $A^{1 *}$, Danlami $S^{3}$, Yunusa $U^{2}$ and Umar LB $^{2}$ \\ ${ }^{1}$ Department of Nursing Sciences, Medical-Surgical Unit, Bayero University, Nigeria \\ ${ }^{2}$ Department of Nursing Sciences, Community-Health Nursing Unit, Bayero University, Nigeria \\ ${ }^{3}$ College of Nursing and Midwifery, Nigeria
}

*Corresponding author: Dalhatu A, Department of Nursing Sciences, Medical-Surgical Unit, Bayero University, Nigeria

\begin{abstract}
High-blood pressure episode is a strong independent risk factor for death from heart disease and stroke. It is usually common diagnostic investigations in modern day cardiology especially in high temperate region where its cut across all ages. Hot temperate regions, Nigeria inclusive has recorded appreciable headway in improving the health care outcomes of its populations, in spite of the challenges posed by pervasive poverty, epidemic diseases, and food insecurity. Checking out both communicable and noncommunicable disease is necessary if quality and effective health care service is to be made available in the context of primary healthcare development. This article reviewed relevant literatures related to hypertension, prevalence and its associated risk which were published by journals nationally and internationally. There is increasing evidence in hot temperate regions in which Nigeria is located, that high-blood pressure is becoming a common clinical condition among nearly all population irrespective of their ages. Hypertensive episodes and quality of care is taking a reverse direction in hot temperate zones. This paradigm shift witness in most non communicable disease necessitates acute response by cardiologist to decreasing the effect of cardiac death and stroke. Hypertension control requires expertise and on-going sensitization programs in hot temperate zones to improving health outcome. The probable challenges faced by the treatment of hypertensive episode, focuses on the progress made toward the health-related Sustainable Development Goals (SDGs) in Nigeria, and the need to establish more robust health systems. We therefore, recommend implementation of sustainable, aggressive and measurable population-based programs for high blood pressure awareness, prevention, treatment, and control as well as investments in improved surveillance and program-relevant research to provide the evidence base for policy development and effective hypertension prevention and control.
\end{abstract}

Keywords: Hypertension; Health; Epidemic; Services; Tropic

\section{Introduction}

High blood pressure, also known as hypertension, has been tagged as the "largest epidemic ever known to mankind" by the medical and scientific world [1]. High-blood pressure has been reported based on available clinical evidences that, is a powerful independent risk factor for death from heart disease and stroke [2]. However, epidemiological data survey to guide decision making and evaluating cardiovascular risk is limited in Nigeria or in the tropics. Studies from around the world, provide important and disturbing findings in both epidemiology and clinical outcomes [3]. It is a common knowledge in the tropics that, overall hypertension prevalence was projected to be between $10 \%-15 \%$, and prevalence rates is increasing at faster rate as high as $30 \%-32 \%$ in middleincome urban and some rural areas [3]. Notably, hypertension awareness, treatment, and control rates is declining to a lower level. Cardiovascular risk has been by far the most common clinical sequel following hypertensive episodes.

In most temperate settings, hypertension control has been projected to be receiving low attention, coupled with low experiences or lack of expertise compounded the increase risk of hypertensive crisis. Health-system researches showed that rapid urbanization and transition from agrarian life to low economic buoyancy of individuals living in the tropics continue to stimulate increases in average blood pressure levels and prevalence of hypertension. Sadly enough, the actual impact of high blood pressure in the tropic remains largely unmeasured [1]. Observational studies documented associated disability from stroke, heart failure, and kidney failure and death in this region. Previous reports documented that success in checking out the 
health burden of hypertensive crisis depends on measurable health education program, mass awareness and population-based program [4]. Experts in hypertension management reveals that critical care is needed to improved primodial, primary, secondary and tertiary levels of hypertension prevention strategy in the tropic. Globally, cardiovascular disease accounts for about 17 million deaths a year, with high blood pressure being the major cause, largely by causing heart disease and stroke [5].

\section{Health burden of hypertension in the tropic}

World Health Organization [6], documented low prevalence of people with hypertension and had access to effective treatment. A recent systematic review of hypertension studies for tropical zones found awareness on hypertension ranged from $10 \%$ in Ghana to only 8\% in Gabon [7]. Observationally, there is substantial evidence that hypertension is highly prevalent among poorer groups and that they are less likely to have access to effective treatment. Evidently, a national survey in Nigeria found that $23 \%$ of patients and their families fell below a US $\$ 1$-a-day poverty line, this hinted that a good number could not afford dietary modification strategy and could not afford effective management [8].

\section{Clinical etiology of hypertension in the tropic}

a. High blood pressure often occurs on its own, especially when there is family history.

b. Genetic predisposition

c. Metabolic syndrome particularly associated with type -2 diabetes.

d. Sedentary lifestyle

e. Social factors; for example, cigarette smoking this usually constrict blood vessel thereby decreasing peripheral resistance, alcohol consumption, high cholesterol level particularly low-density fat etc.

f. Physical factor; in the tropic region, it is usually hypothesized that high temperature stimulates rennin production by the kidney, which in turn stimulate rennin-angiotensin mechanism. This in turn lowers peripheral resistance leading increase in blood pressure.

g. Poverty

h. Obesity

i. Unhealthy nutrition

\section{Early clinical features of hypertension in the tropic}

a. Asymptomatic

b. Seen as disease of the west, despite the growing body of evidence that hypertension is commoner in the tropic than anywhere in the world.

c. Elevation in systolic and diastolic blood pressure.

d. Nausea and vomiting

\section{Investigations}

a. Blood pressure monitoring

b. Based on clinical sign and symptoms.

c. ECG

d. Cardiac enzymes assay

\section{Treatment/prevention strategy}

a. Primodial prevention; this involves the use of preventive strategy that aimed at preventing the triggers of hypertension in the tropics from getting in contact with the individuals.

b. Primary prevention; this involves creation of measurable population-based programmed to checking the episodes of hypertensive episodes. For example, keep fitting exercise, consumption of healthy diet and dietary modification, national policies.

c. Secondary prevention; this involves the use of hypotensive agents and diuretics.

d. Tertiary prevention; this involves rehabilitation mechanism and prevention of organ damage.

\section{Recommendation and way forward}

The reason for recommendation could be linked to asymptomatic nature of hypertensive episodes which make the patient unwilling to take medications daily for the prevention of complications.

a. Global health policy priorities should focus more on noncommunicable diseases such as hypertension.

b. Regular monitoring and reliable drug supply.

c. A DASH-type diet to stay healthy; DASH stands for dietary approaches to stop hypertension. This includes lots of whole grain products, fish, poultry and nuts. It is rich in potassium, magnesium and calcium, as well as protein and fiber and limits sodium intake to 1,500 milligrams per day.

d. Foods that are rich in potassium like bananas, sweet potatoes, broccoli, lima beans and peas are heart healthy options that can help get blood pressure under control.

e. Researchers believe that potassium counteracts the effects of sodium and reduces blood pressure.

f. A recent study also suggests that foods high in nitrates like beets can be helpful in lowering blood pressure, probably since, nitrates reduces blood pressure by widening blood vessels, reducing the blood pressure and increasing blood flow [9].

g. Beet root juice contains nitrates that are converted to nitric oxide; this gas dilates blood vessels and helps lower blood pressure.

h. Egg whites have a protein that has been shown in a recent 
study to significantly reduce blood pressure.

i. Sesame and rice bran oils, which can be mixed, contain fatty acids and antioxidants that are believed to help reduce blood pressure.

j. Dark chocolate contains flavanols that are antioxidants believed to increase blood vessel elasticity which helps to reduce blood pressure; patients should consume an ounce or less a day, and the chocolate treat should contain at least 70 percent cocoa.

\section{Conclusion}

Hypertensive crisis in the tropic is rapidly taking a new outlook and is changing by pervasive poverty, high temperature and lack of expertise in the management of hypertensive conditions that make preventable deaths, and permanent disability from hypertensive episodes still a perennial healthcare agenda in the region.

\section{References}

1. Addo J, Smeeth L, Leon D (2007) Hypertension in sub-Saharan Africa: a systematic review Hypertension 50: 1012-1018.
2. Cooper RS, Amoah AG, Mensah GA (2003) High blood pressure: the foundation for epidemic cardiovascular disease in African populations. Journal of Ethnicity and Disease 13(2): S48-52.

3. Lim SS, Vos T, Flaxman AD, et al. (2012) A comparative risk assessment of burden of disease and injury attributable to 67 risk factors and risk factor clusters in 21 regions, 1990-2010: a systematic analysis for the Global Burden of Disease Study 2010. Lancet 380: 2224-2260.

4. Lawes CMM, Vander HS, Rodgers A (2008) Global burden of blood pressure-related disease. Lancet 371: 1513-1518.

5. World Health Organization (WHO 2012) Global Status Report on Non communicable Diseases.

6. World Health Organization (WHO 2013) A Global Brief on Hypertension.

7. Lloyd Sherlock P, Minicuci N, Beard J, Ebrahim S, Chatterji S (2014) Hypertension among older adults in low and middle income countries: prevalence, awareness and control. Int J Epidemiol.

8. Kayima J, Wanyenze R, Katamba A, Leontsini E, Nuwaha F (2013) Hypertension awareness, treatment and control in Africa: a systematic review. BMC Cardiovasc Disord 13: 54.

9. Peter L, Shah E (2014) Is hypertension the new HIV epidemic? International Journal of Epidemiologyije, oxfordjournals.org.

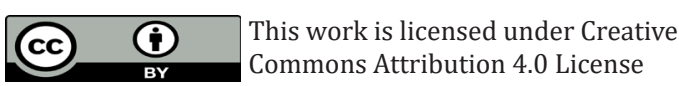

To Submit Your Article Click Here:

Submit Article

DOI: $10.32474 /$ GJAPM.2019.02.000135

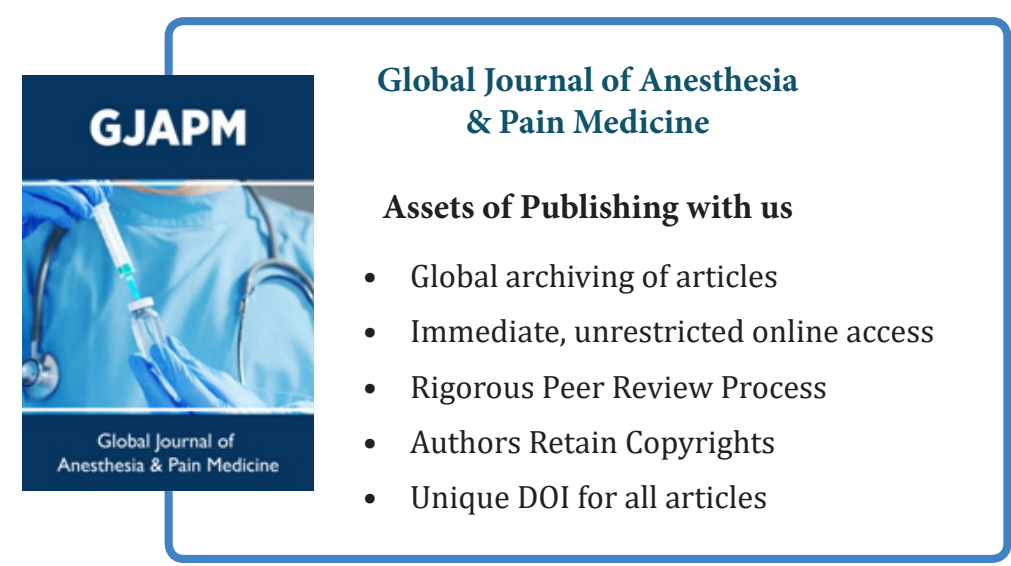

